\title{
Quantum Correlation of Light Mediated by Gravity
}

\author{
Haixing Miao, ${ }^{1, *}$ Denis Martynov, ${ }^{1, \dagger}$ Huan Yang, ${ }^{2,3, \$}$ and Animesh Datta ${ }^{4}, \S$ \\ ${ }^{1}$ School of Physics and Astronomy, and Institute for Gravitational Wave Astronomy, \\ University of Birmingham, Edgbaston, Birmingham B15 2TT, United Kingdom \\ ${ }^{2}$ Perimeter Institute for Theoretical Physics, Waterloo, ON N2L2Y5, Canada \\ ${ }^{3}$ University of Guelph, Guelph, ON N2L3G1, Canada \\ ${ }^{4}$ Department of Physics, University of Warwick, Coventry CV4 7AL, United Kingdom
}

\begin{abstract}
We propose to explore the quantum nature of gravity using the correlation of light between two optomechanical cavities, and the quantumness of the correlation is witnessed by squeezing. As long as the gravity between the end mirrors of two cavities is quantum in the Newtonian limit, we show that the squeezing is always nonzero and monotonically increases as the mechanical property of the mirrors is improved. The proposed scheme provides a new pathway for testing the quantum nature of gravity systematically with tabletop experiments.
\end{abstract}

Introduction - Constructing a consistent and verifiable quantum theory of gravity has been a longstanding challenge of modern physics [1-3], which is partially due to the difficulty in experimentally observing quantum effects of gravity. This, to certain extents, motivates some theoretical models that treat gravity as a fundamental classical entity [411] or being emerged from yet unknown underlying microphysics [12-15]. Experimentally probing the quantum nature of gravity is therefore essential for providing hints towards constructing the correct model $[16,17]$. Recently, two experimental proposals have been made to demonstrate gravityinduced quantum entanglement between two mesoscopic test masses [18, 19], motivated by an early suggestion of Feynman [20]. Both involve two matter-wave interferometers located close to each other such that their test masses can be entangled through the gravitational interaction. Whether gravity-mediated entanglement in the Newtonian limit establishes the quantumness of gravity or not has been debated [2125], because the radiative degrees of freedom-the graviton, are not directly probed in these experiments. Nonetheless, such experiments are important steps towards understanding gravity in the quantum regime [26-31].

The challenge of demonstrating gravity-induced entanglement is achieving a very low thermal decoherence rate, and is beyond what can be achieved with the state-of-the-art instruments, as illustrated in the Appendix A. In this paper, we propose a tabletop optomechanical experiment to explore gravitymediated quantum correlation of light. The strength of the correlation is quantified by squeezing, which is non-classical according to the Glauber-Sudarshan distribution function [3234]. The setup is shown schematically in Fig. 1. Two optomechanical cavities are placed close to each other with their end mirrors interacting through gravity. In contrast to the singlephoton nonlinear regime studied by Balushi et al. [35], we consider the linear regime with the cavity driven by a coherent laser field. The quantum correlation is inferred by squeezing of the outgoing field of the cavity A conditional on the homodyne measurement of the outgoing field of B.

\footnotetext{
* haixing@star.sr.bham.ac.uk

$\dagger$ dmartynov@star.sr.bham.ac.uk

¥ hyang@perimeterinstitute.ca

§animesh.datta@warwick.ac.uk
}

If the gravitational interaction between two mirrors is quantum in the Newtonian limit, namely,

$$
\hat{H}_{A B}=-\frac{G m_{A} m_{B}}{\left|\hat{q}_{A}-\hat{q}_{B}\right|},
$$

we will show such a conditional squeezing is always nonzero. Observing a sizeable squeezing however requires the optomechanical cavities to be quantum radiation pressure limited, in which case the squeezing can be approximately as

$$
\begin{aligned}
\mathcal{S} & =10 \log _{10}\left[1+\left(\frac{2 Q_{m} G \rho}{\omega_{m}^{2}}\right)^{2}\right] \\
& \approx 2 \mathrm{~dB}\left(\frac{0.5 \mathrm{~Hz}}{\omega_{m} / 2 \pi}\right)^{4}\left(\frac{Q_{m}}{3 \times 10^{6}}\right)^{2}\left(\frac{\rho}{20 \mathrm{~g} / \mathrm{cm}^{3}}\right)^{2} .
\end{aligned}
$$

It only depends on the gravitational constant $G$, material density $\rho$, mechanical frequency $\omega_{m}$, and quality factor $Q_{m}$.

The statistical uncertainty of the measurement will affect the squeezing signal. Fortunately, because the system is in a steady state, the signal-to-noise ratio (SNR) increases as the measurement time $\tau$. Achieving a unity SNR requires

$$
\tau \approx 1 \text { year }\left(\frac{\omega_{m} / 2 \pi}{0.5 \mathrm{~Hz}}\right)^{3}\left(\frac{3 \times 10^{6}}{Q_{m}}\right)\left(\frac{20 \mathrm{~g} / \mathrm{cm}^{3}}{\rho}\right)^{2} .
$$

Both $\mathcal{S}$ and $\tau$ scale rapidly with $\omega_{m}$, and low-frequency mechanical oscillators are therefore preferable.

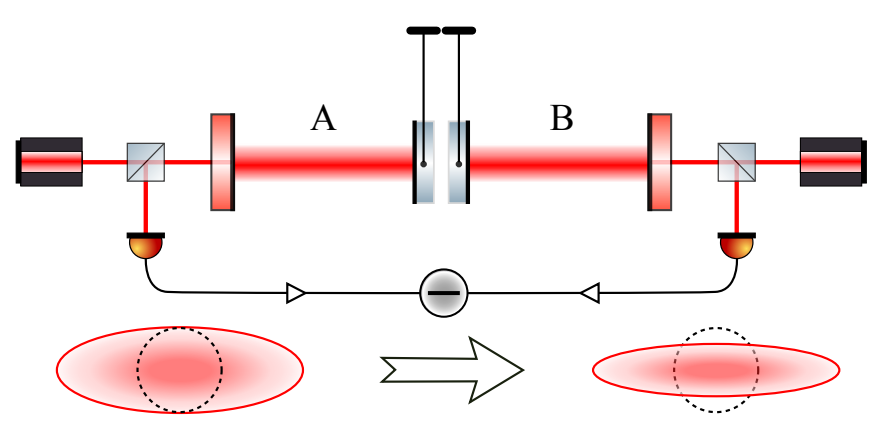

FIG. 1. Schematics showing the proposed experimental setup (the local oscillator for the homodyne detection of the outgoing field of $\mathrm{B}$ is not shown). Squeezing of the outgoing field of A conditional on measuring that of B manifests the gravity-mediated quantum correlation. Dashed circle denotes the vacuum level. 
There are several optomechanical experiments that have achieved the quantum radiation pressure limited regime but with high-frequency mechanical oscillators [36-41] and in particular, Ref. [40] reported a steady-state entanglement between light mediated by a mechanical oscillator. Advancing these experimental techniques towards low frequencies, also an effort in the gravitational-wave community [42-45], is the key to measure the gravity-mediated quantum correlation.

Dynamics - The derivation of Eq. (2) follows the lineardynamics analysis in quantum optomechanics [46, 47]: Solving the linear Heisenberg equations of motion for dynamical variables, which are the mirror position and quadratures of the outgoing optical fields, and representing them in terms of external fields, which are the ingoing optical fields and the thermal bath field.

The total Hamiltonian of the system is $\hat{H}_{\text {tot }}=\hat{H}_{A}+\hat{H}_{B}+\hat{H}_{A B}$. The individual cavity is quantified by the standard linearised optomechanical Hamiltonian, which describes the radiationpressure coupling between the optical field and the centre of mass motion of the mirror (mechanical degree of freedom). The interaction part of $\hat{H}_{A}$ for cavity A is (similarly for B):

$$
\hat{H}_{A}^{\text {int }}=\hbar \omega_{q} \hat{X}_{A} \hat{Q}_{A} .
$$

We denote $\hat{X}_{A}$ as the amplitude quadrature of the cavity mode, which is conjugate to the phase quadrature $\hat{Y}_{A}:\left[\hat{X}_{A}, \hat{Y}_{A}\right]=i$, and $\hat{Q}_{A}$ as the mirror position $\hat{q}_{A}$ normalised with respect to its zero-point motion $\sqrt{\hbar /\left(2 m \omega_{m}\right)}$. The parameter $\omega_{q}$ describes the optomechanical coupling strength:

$$
\omega_{q} \equiv \sqrt{\frac{2 P_{\mathrm{cav}} \omega_{0}}{m c L \omega_{m}}},
$$

which depends on the intra-cavity optical power $P_{\text {cav }}$, the laser frequency $\omega_{0}$, the mirror mass $m$, and the cavity length $L$.

Up to the second-order of the mirror position, the nontrivial interaction part of $\hat{H}_{A B}$ in Eq. (1) is

$$
\hat{H}_{A B}=\hbar \frac{\omega_{g}^{2}}{\omega_{m}} \hat{Q}_{A} \hat{Q}_{B}
$$

Here we have assumed two mirrors having the same mechanical frequency and mass $m_{A}=m_{B}=m$. The characteristic gravitational interaction frequency $\omega_{g}$ is equal to $\sqrt{G m / d^{3}}$ when the two mirrors have a mean separation $d$ much larger than their size, which is the case for mesoscopic levitating masses considered in Refs. [18, 19, 41, 48]. For macroscopic test mass mirrors of gram or kilogram scale, their separation can be made comparable to their size (yet not affected by e.g. the Casimir force), and we have

$$
\omega_{g}=\sqrt{\Lambda G \rho},
$$

which does not explicitly depend on the mirror mass. The form factor $\Lambda$ is determined by the geometry of two mirrors. It is $\pi / 3$ for two spheres with the mean separation equal to twice of the radius, and we assume $\Lambda=2.0$ throughout the paper, which is a good approximation for two closely-located disks with the radius being 1.5 times its thickness (see Appendix B for details).
Solving the Heisenberg equations of motion results in the following frequency domain input-output relation for cavity A (similarly for cavity B):

$$
\begin{aligned}
& \hat{X}_{A}^{\text {out }}(\omega)=\hat{X}_{A}^{\text {in }}(\omega), \\
& \hat{Y}_{A}^{\text {out }}(\omega)=\hat{Y}_{A}^{\text {in }}(\omega)+\sqrt{2 / \gamma} \omega_{q} \hat{Q}_{A}(\omega),
\end{aligned}
$$

where we have assumed that the cavity bandwidth $\gamma$ is much larger than the frequency of interest so that the cavity mode can be adiabatically eliminated, cf. Eq. (2.68) of Ref. [46]. The position of mirror A satisfies

$$
\hat{Q}_{A}=\chi_{q q}\left[\sqrt{\gamma / 2} \omega_{q} \hat{X}_{A}^{\text {in }}-\left(\omega_{g}^{2} / \omega_{m}\right) \hat{Q}_{B}+2 \sqrt{\gamma_{m}} \hat{Q}_{A}^{\text {th }}\right] .
$$

Here $\chi_{q q} \equiv-\omega_{m} /\left(\omega^{2}-\omega_{m}^{2}+i \gamma_{m} \omega\right)$ is the susceptibility with the mechanical damping rate $\gamma_{m} \equiv \omega_{m} / Q_{m}$; $\hat{Q}^{\text {th }}$ is the normalised thermal Langevin force according to the fluctuationdissipation theorem $[49,50]$, and its double-sided spectral density is equal to $\bar{n}_{\text {th }}+(1 / 2)$ with the thermal occupation number $\bar{n}_{\text {th }} \equiv k_{B} T /\left(\hbar \omega_{m}\right)$ in the high-temperature limit.

The final input-output relation involving both cavities is

$$
\left[\begin{array}{c}
\hat{X}_{A}^{\text {out }} \\
\hat{Y}_{A}^{\text {out }} \\
\hat{X}_{B}^{\text {out }} \\
\hat{Y}_{B}^{\text {out }}
\end{array}\right]=\left[\begin{array}{cccc}
1 & 0 & 0 & 0 \\
\mathcal{K} & 1 & \mathcal{G} & 0 \\
0 & 0 & 1 & 0 \\
\mathcal{G} & 0 & \mathcal{K} & 1
\end{array}\right]\left[\begin{array}{c}
\hat{X}_{A}^{\text {in }} \\
\hat{Y}_{A}^{\text {in }} \\
\hat{X}_{B}^{\text {in }} \\
\hat{Y}_{B}^{\text {in }}
\end{array}\right]+\left[\begin{array}{cc}
0 & 0 \\
\alpha & \beta \\
0 & 0 \\
\beta & \alpha
\end{array}\right]\left[\begin{array}{c}
\hat{Q}_{A}^{\text {th }} \\
\hat{Q}_{B}^{\text {th }}
\end{array}\right] .
$$

Here $\mathcal{K} \equiv-4 \omega_{q}^{2} \chi_{q q} / \gamma$ quantifies the correlation between the amplitude quadrature and the phase quadrature in the individual cavity and is responsible for the optomechanical squeezing [51-55]. The two parameters $\alpha \equiv 2 \sqrt{2 \gamma_{m} / \gamma} \omega_{q} \chi_{q q}$ and $\beta \equiv \alpha \chi_{q q}\left(\omega_{g}^{2} / \omega_{m}\right)$ quantify the output response to the thermal force noise. As illustrated in Fig. 2, the dimensionless parameter $\mathcal{G}$ quantifies the mutual correlation between two cavities and is defined as $\mathcal{G} \equiv 4 \omega_{q}^{2} \omega_{g}^{2} \chi_{q q}^{2} /\left(\gamma \omega_{m}\right)$. Its magnitude reaches the maximum at the mechanical frequency:

$$
\left|\mathcal{G}\left(\omega_{m}\right)\right|=2 C Q_{m}\left(\frac{\omega_{g}}{\omega_{m}}\right)^{2} .
$$

The optomechanical cooperativity defined as

$$
C \equiv \frac{2 \omega_{q}^{2}}{\gamma \gamma_{m}}
$$

is proportional to the number of intra-cavity photons [47]. The fact that $|\mathcal{G}|$ is proportional to $C$ shows that the optomechanical interaction coherently enhances the correlation by amplifying the quantum fluctuation of light.

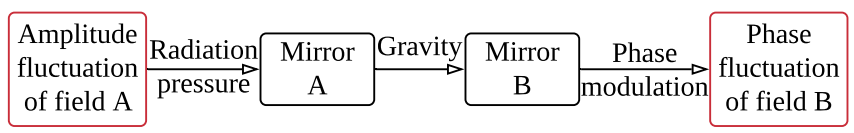

FIG. 2. A flow chart illustrating the physical meaning of $\mathcal{G}$ introduced in the input-output relation Eq. (11). 
Quantum correlation and conditional squeezing - Notice that the correlation reaches the maximum around the mechanical frequency within a narrow frequency bandwidth defined by $\gamma_{m}$. We can therefore focus on the quadratures of the outgoing fields around $\omega_{m}$ with a bandwidth $\Delta \omega$ comparable to $\gamma_{m}$ (or the measurement time comparable to the damping time $\left.\tau_{m} \equiv 2 \pi Q_{m} / \omega_{m}\right)$. The corresponding normalised quadrature operators are defined as

$$
\hat{X} \equiv \sqrt{\Delta \omega / \pi} \hat{X}^{\text {out }}\left(\omega_{m}\right), \quad \hat{y} \equiv \sqrt{\Delta \omega / \pi} \hat{Y}^{\text {out }}\left(\omega_{m}\right) .
$$

They satisfy $\left[\hat{\mathcal{X}}, \hat{\mathcal{Y}}^{\dagger}\right]=2 i$, where we have approximated the Dirac delta function $\delta(0)$ as $1 / \Delta \omega$. With such a normalisation, the uncertainty of $\hat{X}$ or $\hat{y}$ for the vacuum or coherent state is equal to 1.

Due to the quantum correlation, the uncertainty of the amplitude quadrature of A can be reduced after we measure the phase quadrature of $\mathrm{B}$. The conditional uncertainty is obtained by minimising the residue over the filtering function $\mathcal{F}$ :

$$
\begin{aligned}
\sigma_{\mathcal{X}}^{\mathrm{cond}} & =\min _{\mathcal{F}} \operatorname{Tr}\left[\hat{\varrho}\left(\hat{\mathcal{X}}_{A}-\mathcal{F} \hat{\mathcal{Y}}_{B}\right)^{2}\right]=\sigma_{\mathcal{X} X}-\frac{\left|\sigma_{X y}\right|^{2}}{\sigma_{y y}} \\
& =1-\frac{|\mathcal{G}|^{2}}{1+|\mathcal{K}|^{2}+|\mathcal{G}|^{2}+\left(2 \bar{n}_{\mathrm{th}}+1\right)\left(|\alpha|^{2}+|\beta|^{2}\right)},
\end{aligned}
$$

where we define the variance $\sigma_{\mathcal{X} X} \equiv \operatorname{Tr}\left[\varrho \hat{\mathcal{X}}_{A} \hat{\mathcal{X}}_{A}^{\dagger}\right]$ (similar for $\sigma_{y y}$ of $\left.\hat{\mathcal{Y}}_{B}\right)$, and the covariance $\sigma_{\mathcal{}} \equiv \operatorname{Tr}\left[\varrho\left(\hat{\mathcal{X}}_{A} \hat{\mathcal{Y}}_{B}^{\dagger}+\right.\right.$ $\left.\hat{\mathcal{Y}}_{B}^{\dagger} \hat{\mathcal{X}}_{A}\right) / 2$ ] with $\hat{\varrho}$ being the density matrix. In obtaining the above result, we have used the fact that the ingoing optical field is in the vacuum state because the coherent amplitude is absorbed by the coupling rate $\omega_{q}[46,47]$. The corresponding optimal Wiener filter is given by $\mathcal{F}_{\text {opt }}=\sigma_{\mathcal{X} y} / \sigma_{y y}=$ $\mathcal{G} /\left[1+|\mathcal{K}|^{2}+|\mathcal{G}|^{2}+\left(2 \bar{n}_{\text {th }}+1\right)\left(|\alpha|^{2}+|\beta|^{2}\right)\right]$.

As we can see from Eq. (15), the conditional uncertainty of $\hat{X}_{A}$ is always smaller than 1 , which implies squeezing. To observe such a conditional squeezing experimentally, the estimation error due to a finite number of measurements needs to be smaller than the squeezing level. According to the standard estimation theory, the unbiased estimator for the conditional uncertainty for a known average is

$$
\sigma_{\mathcal{X} X}^{\text {est }}=\frac{1}{N_{s}} \sum_{k=1}^{N_{s}} \tilde{\sigma}_{\mathcal{X} X}^{\text {cond }}(k)
$$

where $\tilde{\sigma}_{\mathcal{X}}^{\text {cond }}(k)$ is the conditional variance for the $k$-th measurement sample and $N_{s}$ is the total number of samples. In our case, each sample corresponds to a measurement time of the order of the mechanical damping time $\tau_{m}$. For a total measurement time of $\tau$, we have

$$
N_{s} \equiv \frac{\tau}{\tau_{m}}=\frac{\omega_{m} \tau}{2 \pi Q_{m}}
$$

Since $\sum_{k=1}^{N_{s}} \tilde{\sigma}_{\mathcal{X} X}^{\text {cond }}(k)$ follows the chi-squared distribution with $N_{s}$ degrees of freedom, the estimation error is equal to $\sqrt{2 / N_{s}} \sigma_{\mathcal{X} X}^{\text {cond }}$. It needs to be smaller than the squeezing level to achieve a unity SNR, which implies

$$
\sqrt{\frac{2}{N_{s}}} \sigma_{\mathcal{X} \mathcal{X}}^{\text {cond }} \leq \frac{|\mathcal{G}|^{2}}{1+|\mathcal{K}|^{2}+|\mathcal{G}|^{2}+\left(2 \bar{n}_{\mathrm{th}}+1\right)\left(|\alpha|^{2}+|\beta|^{2}\right)} .
$$

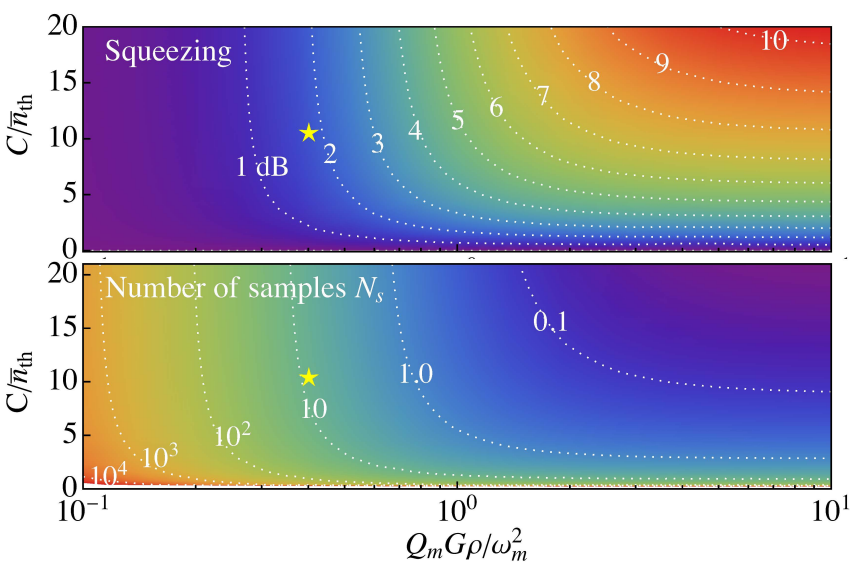

FIG. 3. The top panel shows the squeezing in $\mathrm{dB}$ as a function of two dimensionless parameters: $C / \bar{n}_{\text {th }}$ and $Q_{m} G \rho / \omega_{m}^{2}$. The bottom panel shows the minimum $N_{s}$ needed to achieve a unity signal-tonoise ratio $\left(N_{s}<1\right.$ implies one sample is sufficient), and a small $N_{s}$ does not mean a short measurement time, which is equal to $N_{s}$ times the mechanical damping time. The two stars on the graphs mark the parameters assumed in Eq. (2) and Eq. (3) of the introduction part.

The above condition leads to a requirement on the minimum measurement time $\tau$. For experimentally relevant parameters, we have $\bar{n}_{\text {th }} \gg 1$ and $|\mathcal{K}| \gg 1$, we can approximate the denominator of Eq. (15) and Eq. (18) as $|\mathcal{K}|^{2}+|\mathcal{G}|^{2}+2 \bar{n}_{\text {th }}\left(|\alpha|^{2}+|\beta|^{2}\right)$. The resulting squeezing and also the minimum number of samples are shown in Fig. 3. They only depend on two characteristic dimensionless parameters: $C / \bar{n}_{\mathrm{th}}$, the ratio between the optomechanical cooperativity and the thermal occupation number, and $Q_{m} G \rho / \omega_{m}^{2}$, solely determined by the gravity and the mechanical property of the mirror.

To obtain a sizeable squeezing, we learn from Fig. 3 that first $Q_{m} G \rho / \omega_{m}^{2}$ needs to be large, which implies high-qualityfactor, low-frequency test mass mirrors, and second the cooperativity shall be much larger than the mean thermal occupation number, namely,

$$
C \gg \bar{n}_{\text {th }} \text {. }
$$

This corresponds to the quantum radiation pressure limited regime in optomechanics [47]. In such a regime, the squeezing and minimum number of samples turn out to become independent of the optical property and only depend on the mechanical property. In particular, we have

$$
\sigma_{\mathcal{X} X}^{\text {cond }} \approx \frac{1}{1+|\mathcal{G} / \mathcal{K}|^{2}}=\frac{1}{1+\left(2 Q_{m} G \rho / \omega_{m}^{2}\right)^{2}},
$$

which, written in terms of $\mathrm{dB}$, gives rise to Eq. (2) shown in the introduction. The minimum number of samples $N_{s}$ to achieve a unity SNR can be approximated as

$$
N_{s} \approx 1+4|\mathcal{K} / \mathcal{G}|^{2} \approx\left(\frac{\omega_{m}^{2}}{Q_{m} G \rho}\right)^{2} .
$$

The second approximation is satisfied for those parameter values assumed in Eq. (3) where we have shown the equivalent minimum measurement time. 
Conclusions and discussions - To summarise, our approach for probing the quantum nature of gravity takes advantage of new advancements in quantum optomechanical experiments. It is complimentary to other approaches based upon matter-wave interferometers. In general, achieving a sizeable squeezing requires quantum radiation pressure limited systems with high-quality-factor, low-frequency mechanical test mass mirrors. Even though the squeezing signal does not explicitly depend on the size of the test mass mirror, having a low mechanical frequency usually implies macroscopic test masses. For illustration, we provide a possible set of sample parameters to reach $C / \bar{n}_{\text {th }}$ of the order of 10 implicitly assumed in Eq. (2) for $\omega_{m} /(2 \pi)=0.5 \mathrm{~Hz}$ and $Q_{m}=3 \times 10^{6}$ :

$$
\frac{C}{\bar{n}_{\mathrm{th}}} \approx 10\left(\frac{1 \mathrm{~g}}{m}\right)\left(\frac{P_{\mathrm{cav}}}{2 \mathrm{~kW}}\right)\left(\frac{\text { Finesse }}{4000}\right)\left(\frac{300 \mathrm{~K}}{T}\right),
$$

which corresponds to a suspended high-finesse cavity with a gram-scale test mass mirror at room temperature, close to what has been achieved by the MIT group [56]. The gravity experiments with milligram test masses $[57,58]$ can be promising if pushed to the low-frequency regime.

Let us consider the consequence of different outcomes of the measurement that we propose. If we do not detect a predicted level of squeezing after a careful calibration of the system, it will imply that the assumption on the gravity sector is invalid, cf. Eq. (1), as the quantum aspects of the optomechanical interactions have already been established experimentally. One compelling possibility then is that gravity is classical, so that it does not appear in the quantum interaction Hamiltonian. If we do observe a non-zero squeezing, we will be able to rule out classical models of gravity, in particular the SchrödingerNewton (SN) type of classical gravity models - the gravity is sourced by the expectation value of quantum matters [4-11], which does not lead to quantum correlation. This is because the corresponding SN two-body interaction for the optomechanical setup would be, cf. Eq. (27) of Ref. [9],

$$
\hat{H}_{\mathrm{AB}}^{\mathrm{SN}}=\hbar \frac{\omega_{g}^{2}}{2 \omega_{m}}\left(\left\langle\hat{Q}_{A}\right\rangle \hat{Q}_{B}+\hat{Q}_{A}\left\langle\hat{Q}_{B}\right\rangle\right)
$$

According to Eq. (10), the quantum part of $\left\langle\hat{Q}_{A}\right\rangle$ or $\left\langle\hat{Q}_{B}\right\rangle$ is zero, as the expectation value of the quantum fluctuation $\hat{X}_{A}^{\text {in }}$ is zero. For future study, it would be interesting also to explore the predictions of emergent gravity models [12-15] on the conditional squeezing level in this proposed optomechanical setup.

Acknowledgements - We would like to thank Yanbei Chen, Chris Collins, Bassam Helou, and Dominic Branford for fruitful discussions, and Joe Bentley for proofreading the manuscript. H.M. is supported by UK STFC Ernest Rutherford Fellowship (Grant No. ST/M005844/11). H.Y. is supported by the Natural Sciences and Engineering Research Council of Canada, and by Perimeter Institute for Theoretical Physics. D.M. acknowledges the support from the Institute for Gravitational-wave Astronomy at University of Birmingham. A.D. is supported, in part, by the UK EPSRC (EP/K04057X/2), and the UK National Quantum Technologies Programme (EP/M01326X/1, EP/M013243/1).

\section{Appendix A: Condition for realising gravity-mediated entanglement}

Here we try to derive the general condition for achieving entanglement between the outgoing fields of two cavities. The entanglement measure can be derived from their total covari-

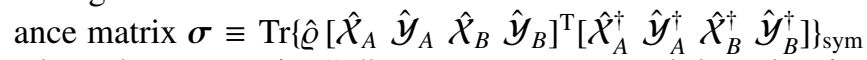
where the superscript " $T$ " means transpose and the subscript "sym" means symmetrisation: $\operatorname{Tr}[\hat{\varrho} \hat{\mathcal{X}} \hat{\mathcal{Y}}]_{\mathrm{sym}} \equiv \operatorname{Tr}\left[\hat{\varrho}\left(\hat{\mathcal{X}} \hat{y}^{\dagger}+\right.\right.$ $\left.\left.\hat{y}^{\dagger} \hat{x}\right) / 2\right]$, more explicitly,

$$
\sigma \equiv\left[\begin{array}{cc}
\sigma_{A} & \sigma_{A B} \\
\sigma_{A B}^{\mathrm{T}} & \sigma_{B}
\end{array}\right]
$$

The diagonal components $\sigma_{A}=\sigma_{B}$ are

$$
\sigma_{A}=\left[\begin{array}{cc}
1 & \mathcal{K}^{*} \\
\mathcal{K} & 1+|\mathcal{K}|^{2}+|\mathcal{G}|^{2}+\left(2 \bar{n}_{\text {th }}+1\right)\left(|\alpha|^{2}+|\beta|^{2}\right)
\end{array}\right] .
$$

The off-diagonal one, describing the cross correlation, is

$$
\sigma_{A B}=\left[\begin{array}{cc}
0 & \mathcal{G} \\
\mathcal{G} & 0
\end{array}\right] .
$$

All the above quantities $\mathcal{K}, \mathcal{G}, \alpha$ and $\beta$ are referring to their values at $\omega_{m}$, in particular,

$$
\mathcal{K}\left(\omega_{m}\right)=-2 i C, \quad \alpha\left(\omega_{m}\right)=2 i \sqrt{C}
$$

Note that $\mathcal{K}\left(\omega_{m}\right)$ is complex and it leads to the complex squeezing, which is unaccessible with the standard homodyne detection $[54,55]$. That is why the noise ellipse of A illustrated in Fig. 1 shows no correlation between the amplitude quadrature and the phase quadrature of $\mathrm{A}$.

The figure of merit for quantifying such a bipartite Gaussian entanglement is the so-called logarithmic negativity $\mathcal{E}_{N}[59$, 60], which is defined as

$$
\mathcal{E}_{N}=\max \left\{-(1 / 2) \ln \left[\left(\Sigma-\sqrt{\Sigma^{2}-4 \operatorname{det} \sigma}\right) / 2\right], 0\right\},
$$

where $\Sigma \equiv \operatorname{det} \sigma_{A}+\operatorname{det} \sigma_{B}-2 \operatorname{det} \sigma_{A B}$. A nonzero $\mathcal{E}_{N}$ implies the existence of entanglement. In our case, the first term is equal to

$$
-\ln \left[\sqrt{1+|\mathcal{G}|^{2}+\left(2 \bar{n}_{\mathrm{th}}+1\right)\left(|\alpha|^{2}+|\beta|^{2}\right)}-|\mathcal{G}|\right] .
$$

Having it larger than zero requires

$$
\left(2 \bar{n}_{\mathrm{th}}+1\right)\left(|\alpha|^{2}+|\beta|^{2}\right)<2|\mathcal{G}| .
$$

When using the fact that $|\alpha| \gg|\beta|$ and $\bar{n}_{\text {th }} \gg 1$, we arrive at the following condition:

$$
\gamma_{m} k_{B} T \leq \hbar G \rho
$$

As an order of magnitude, it implies

$$
\frac{T}{Q_{m}} \leq 3.0 \times 10^{-18} \mathrm{~K}\left(\frac{0.5 \mathrm{~Hz}}{\omega_{m} / 2 \pi}\right)\left(\frac{\rho}{20 \mathrm{~g} / \mathrm{cm}^{3}}\right) .
$$


This requirement is beyond what we can achieve with the state-of-the-art instruments, and needs further experimental efforts. Note that a related analysis of steady-state Gaussian entanglement in the case of two levitating nanobeads has also been presented by Qvarfort et al. [48].

The above requirement Eq. (A8) turns out to be equally applicable to the free-mass case with the resonant frequency $\omega_{m} \rightarrow 0$, as $\omega_{m}$ does not appear explicitly in the equation. We consider the standard thermal decoherence model. The corresponding master equation for the density matrix $\varrho$ of the two test masses takes the following diffusive form:

$$
\dot{\hat{\varrho}}(t)=\frac{i}{\hbar}\left[\hat{\varrho}(t), \hat{H}_{A B}\right]-\frac{2 m \gamma_{m} k_{B} T \delta x_{q}^{2}}{\hbar^{2}} \sum_{j=A, B}\left[\hat{Q}_{j},\left[\hat{Q}_{j}, \hat{\varrho}(t)\right]\right],
$$

where $\delta x_{q}$ is the characteristic length scale and is equal to the Standard Quantum Limit (SQL) [61] for Gaussian states and the size of the quantum superposition for non-Gaussian states. For the quantum entanglement to survive in the presence of the thermal decoherence, we require the interaction rate to be larger than the decoherence rate:

$$
\frac{\left\|\hat{H}_{A B}\right\|}{\hbar} \geq \frac{2 m \gamma_{m} k_{B} T \delta x_{q}^{2}}{\hbar^{2}}
$$

where $\left\|\hat{H}_{A B}\right\|$ is the norm that quantifies the magnitude of the gravitational-interaction energy when $\mathrm{A}$ and $\mathrm{B}$ are at the quantum level.

In the case of $\delta x_{q}$ much smaller than the mean separation $d$, we have, according to Eq. (6),

$$
\left\|\hat{H}_{A B}\right\| \approx 2 \Lambda G m \rho \delta x_{q}^{2},
$$

where we have assumed that $\delta x_{q}$ is the same for $\mathrm{A}$ and $\mathrm{B}$. The condition Eq. (A11) leads to Eq. (A8) for $\Lambda$ being the order of 1. Similarly, when $\delta x_{q}$ is much larger than the mean separation $d$, e.g. the non-Gaussian superposition state in the setup using the matter-wave interferometers [18, 19], the corresponding gravitational interaction energy is simply

$$
\left\|\hat{H}_{A B}\right\|=\frac{G m^{2}}{d} .
$$

Eq. (A11) results in

$$
\gamma_{m} k_{B} T \leq \frac{\hbar G m}{2 d \delta x_{q}^{2}}<\frac{\hbar G m}{2 d^{3}} \leq \hbar G \rho,
$$

where in the last inequality we have used the fact that $m / d^{3}$ is at most of the order of the matter density $\rho$. Therefore, regardless whether the two test masses (either being free mass or harmonic oscillator) are prepared in Gaussian states or nonGaussian states, the same requirement universally applies for achieving the gravity-mediated entanglement in the presence of thermal decoherence.

\section{Appendix B: Dependence of $\Lambda$ on the test mass geometry}

Depending on the geometry of the two test masses, the form factor in defining $\omega_{g}$ in Eq. (7) is different. The simplest case

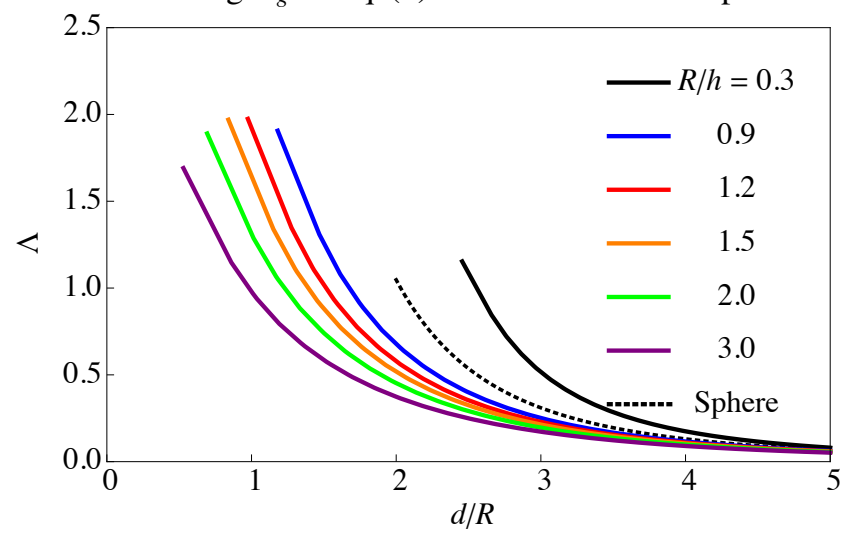

FIG. 4. The form factor $\Lambda$ as a function of distance for different ratios between the radius $R$ and the thickness $h$ of the disk. As a reference, we also show the case of two spheres in dashed line. The lower bound of the distance for different curves are defined by the one when the two disks touch each other.

is having two identical spheres with a uniform density, and $\Lambda=\pi / 3$ when their mean separation is equal to twice of their radius. Here we consider two test masses that have the shape of a disk which is usually the geometry for mirrors of optical cavities. Since there is no analytical expression for the Newtonian force between two disks, we perform numerical integration of the force for disks with different ratios between the radius $R$ and the thickness $h$. We then take the derivative numerically with respect to their mean separation $d$ along the optical axis to obtain $\Lambda$ for different mean separations and the maximum $\Lambda$ is achieved when their surfaces are close to each other with $d$ approximately equal to $h$. Fig. 4 shows the result, and we can see the maximum value of $\Lambda$ for $R / h=1.5$ is around 2.0, which is the one we assumed in the main text.
[1] C. Kiefer, Annalen der Physik (Leipzig) 15, 129 (2006).

[2] R. P. Woodard, Reports on Progress in Physics 72, 126002 (2009).

[3] D. Oriti, ed., Approaches to Quantum Gravity: Toward a New Understanding of Space, Time and Matter (Cambridge University Press, Cambridge, 2009).

[4] C. Møller, Colloques Internationaux CNRS 91, 1 (1962).
[5] L. Rosenfeld, Nuclear Physics 40, 353 (1963).

[6] T. W. B. Kibble, Communications in Mathematical Physics 64, 73 (1978).

[7] S. L. Adler, Journal of Physics A: Mathematical and Theoretical 40, 755 (2007).

[8] S. Carlip, Class. Quant Grav. 25, 154010 (2008). 
[9] H. Yang, H. Miao, D. Lee, B. Helou, and Y. Chen, Phys. Rev. Lett. 110, 170401 (2013).

[10] C. Anastopoulos and B. Hu, New J. Phys. 16, 085007 (2014).

[11] M. Bahrami, A. Großardt, S. Donadi, and A. Bassi, New J. Phys. 16, 115007 (2014).

[12] T. Jacobson, Phys. Rev. Lett. 75, 1260 (1995).

[13] E. Verlinde, Journal of High Energy Physics 2011, 29 (2011).

[14] T. Padmanabhan, Modern Physics Letters A 30, 1540007 (2015).

[15] S. Hossenfelder, Phys. Rev. D 95, 124018 (2017).

[16] R. Howl, L. Hackermüller, D. E. Bruschi, and I. Fuentes, Advances in Physics: X 3, 1383184 (2018).

[17] D. Carney, P. C. E. Stamp, and J. M. Taylor, Class. Quant Grav. 36, 034001 (2019).

[18] S. Bose, A. Mazumdar, G. W. Morley, H. Ulbricht, M. Toroš, M. Paternostro, A. A. Geraci, P. F. Barker, M. S. Kim, and G. Milburn, Phys. Rev. Lett. 119, 240401 (2017).

[19] C. Marletto and V. Vedral, Phys. Rev. Lett. 119, 240402 (2017).

[20] R. P. Feynman, in Chapel Hill Conference Proceedings (1957).

[21] T. Krisnanda, M. Zuppardo, M. Paternostro, and T. Paterek, Phys. Rev. Lett. 119, 120402 (2017).

[22] C. Anastopoulos and B. Hu, arXiv:1804.11315 (2018).

[23] M. J. W. Hall and M. Reginatto, Journal of Physics A: Mathematical and Theoretical 51, 085303 (2018).

[24] A. Belenchia, R. M. Wald, F. Giacomini, E. Castro-Ruiz, Č. Brukner, and M. Aspelmeyer, Phys. Rev. D 98, 126009 (2018).

[25] M. Reginatto and M. J. W. Hall, arXiv:1809.04989 (2018).

[26] L. Diósi, Physics Letters A 120, 377 (1987).

[27] R. Penrose, General Relativity and Gravitation 28, 581 (1996).

[28] A. Bassi, K. Lochan, S. Satin, T. P. Singh, and H. Ulbricht, Rev. Mod. Phys. 85, 471 (2013).

[29] B. Helou, B. J. J. Slagmolen, D. E. McClelland, and Y. Chen, Phys. Rev. D 95, 084054 (2017).

[30] A. Vinante, R. Mezzena, P. Falferi, M. Carlesso, and A. Bassi, Phys. Rev. Lett. 119, 110401 (2017).

[31] A. Bassi, A. Großardt, and H. Ulbricht, Class. Quant Grav. 34, 193002 (2017).

[32] R. J. Glauber, Phys. Rev. 131, 2766 (1963).

[33] E. C. G. Sudarshan, Phys. Rev. Lett. 10, 277 (1963).

[34] U. M. Titulaer and R. J. Glauber, Phys. Rev. 140, B676 (1965).

[35] A. Al Balushi, W. Cong, R. B. Mann, A. A. Balushi, W. Cong, and R. B. Mann, Phys. Rev. A 98, 043811 (2018).

[36] T. P. Purdy, R. W. Peterson, and C. A. Regal, Science 339, 801 (2013).

[37] C. B. Møller, R. A. Thomas, G. Vasilakis, E. Zeuthen, Y. Tsaturyan, M. Balabas, K. Jensen, A. Schliesser, K. Hammerer, and E. S. Polzik, Nature 547, 191 (2017).
[38] M. Rossi, D. Mason, J. Chen, Y. Tsaturyan, and A. Schliesser, Nature 563, 53 (2018).

[39] J. Cripe, N. Aggarwal, R. Lanza, A. Libson, R. Singh, P. Heu, D. Follman, G. D. Cole, N. Mavalvala, and T. Corbitt, arXiv: 1802.10069 (2018).

[40] S. Barzanjeh, E. S. Redchenko, M. Peruzzo, M. Wulf, D. P. Lewis, G. Arnold, and J. M. Fink, arXiv: 1809.05865 (2018).

[41] U. Delić, M. Reisenbauer, K. Dare, D. Grass, V. Vuletić, N. Kiesel, and M. Aspelmeyer, Science, eaba3993 (2020).

[42] M. Punturo, M. Abernathy, F. Acernese, B. Allen, N. Andersson, K. Arun, F. Barone, B. Barr, and Others, Class. Quant. Grav 27, 194002 (2010).

[43] R. X. Adhikari, Rev. Mod. Phys. 86, 121 (2014).

[44] The LIGO Scientific Collaboration, Class. Quant. Grav. 34, 44001 (2017).

[45] H. Yu, D. Martynov, S. Vitale, M. Evans, D. Shoemaker, B. Barr, G. Hammond, S. Hild, J. Hough, S. Huttner, S. Rowan, B. Sorazu, L. Carbone, A. Freise, C. Mow-Lowry, K. L. Dooley, P. Fulda, H. Grote, and D. Sigg, Phys. Rev. Lett. 120, 141102 (2018).

[46] Y. Chen, Journal of Physics B: Atomic, Molecular and Optical Physics 46, 104001 (2013).

[47] M. Aspelmeyer, T. J. Kippenberg, and F. Marquardt, Rev. Mod. Phys. 86, 1391 (2014).

[48] S. Qvarfort, S. Bose, and A. Serafini, arXiv: 1812.09776 (2018).

[49] H. B. Callen and T. A. Welton, Phys. Rev. 83, 34 (1951).

[50] R. Kubo, Reports on Progress in Physics 29, 255 (1966).

[51] H. J. Kimble, Y. Levin, A. B. Matsko, K. S. Thorne, and S. P. Vyatchanin, Phys. Rev. D 65, 022002 (2001).

[52] D. W. C. Brooks, T. Botter, S. Schreppler, T. P. Purdy, N. Brahms, and D. M. Stamper-Kurn, Nature 488, 476 (2012).

[53] A. H. Safavi-Naeini, S. Gröblacher, J. T. Hill, J. Chan, M. Aspelmeyer, and O. Painter, Nature 500, 185 (2013).

[54] T. P. Purdy, P. L. Yu, R. W. Peterson, N. S. Kampel, and C. A. Regal, Phys. Rev. X 3, 031012 (2013).

[55] L. F. Buchmann, S. Schreppler, J. Kohler, N. Spethmann, and D. M. Stamper-Kurn, Phys. Rev. Lett. 117, 030801 (2016).

[56] T. Corbitt, Y. Chen, E. Innerhofer, H. Muller-Ebhardt, D. Ottaway, H. Rehbein, D. Sigg, S. Whitcomb, C. Wipf, and N. Mavalvala, Phys. Rev. Lett. 98, 150802 (2007).

[57] J. Schmöle, M. Dragosits, H. Hepach, and M. Aspelmeyer, Class. Quant Grav 33, 125031 (2016).

[58] N. Matsumoto, M. Sugawara, S. Suzuki, N. Abe, K. Komori, Y. Michimura, Y. Aso, S. B. Cataño-Lopez, and K. Edamatsu, arXiv:1809.05081 (2018).

[59] R. Simon, Phys. Rev. Lett. 84, 2726 (2000).

[60] R. Horodecki, P. Horodecki, M. Horodecki, and K. Horodecki, Rev. Mod. Phys. 81, 865 (2009).

[61] V. B. Braginsky and F. Y. Khalilli, Quantum Measurement (Cambridge University Press, 1992). 